\title{
RECUERDOS, EXPECTATIVAS Y CONCEPCIONES DE LOS ESTUDIANTES PARA MAESTRO SOBRE LA GEOMETRÍA ESCOLAR
}

\author{
Barrantes, Manuel y Blanco, Lorenzo, J. \\ Departamento de Didáctica de las Ciencias Experimentales y de las Matemáticas \\ Universidad de Extremadura. Badajoz \\ barrante@unex.es \\ lblanco@unex.es
}

\begin{abstract}
Resumen. Numerosos trabajos de investigación han puesto de manifiesto la importancia de analizar las concepciones de los estudiantes para profesores sobre las matemáticas y sobre su enseñanza-aprendizaje durante su proceso de formación. Éstas aparecen y se desarrollan durante su etapa escolar y son estables y resistentes a los cambios. Como consecuencia de ello, asumimos que, para aprender a enseñar matemáticas, debemos considerar las exigencias que proceden de las propias concepciones y conocimientos sobre la matemática escolar.

Partiendo de esta premisa hemos desarrollado una investigación con el objetivo de describir y analizar las concepciones sobre la geometría escolar y su enseñanza-aprendizaje de los estudiantes para maestro. Para ello hemos considerado la hipótesis de que los recuerdos y las expectativas de los estudiantes nos dan información para caracterizar sus concepciones en el campo de la geometría y su enseñanza-aprendizaje en primaria.
\end{abstract}

Palabras clave. Concepciones, geometría, formación inicial de profesores, enseñanza de las matemáticas.

Summary. Several studies have shown the importance of prospective teachers' conceptions about mathematics and its teaching-learning during their educational process. They indicate that to learn to teach mathematics we must take into account the demands that originate from our own conceptions of school-level mathematics, since these are stable and resistant to change.

On the basis of this idea, we have developed a study aimed at describing and analysing prospective primary teachers' conceptions about school-level geometry and its teaching-learning. To this end, we considered the hypothesis that the students' memories and expectations provide information with which to characterize prospective primary teachers' conceptions in the field of geometry and its teaching-learning at primary school level.

Keywords. Conceptions, geometry, preservice teachers' knowledge, mathematics teaching.

\section{IMPORTANCIA DE LAS CONCEPCIONES EN LA FORMACIÓN INICIAL DE LOS MAES- TROS}

Numerosos trabajos de investigación han puesto de manifiesto la importancia de analizar las concepciones de los estudiantes para profesores durante su proceso de formación (Koballa y Crawley, 1985; Bromme, 1988; Ernest, 1989, 2000; Thompson 1992; Pajares, 1992; Fennema y Loef, 1992; LLinares, 1993; Mellado, 1996; Azcárate, 1996; Blanco, 1997; Flores, 1998).

El papel de intermediario de los futuros profesores, entre el currículo y los alumnos, no va a ser de un simple transmisor de directrices y sugerencias oficiales, ni siquiera aunque lo intentasen, pues sus concepciones se van a situar en medio de todas las tareas que prepara o realiza en el aula: «El profesor no motiva a ciegas el aprendizaje, como mero operario, sino que interpreta y aplica el curriculum oficial según unos criterios, entre los que destacan sus concepciones.» Carrillo (2000, p. 80).

A este respecto, es conveniente resaltar que algunos de estos trabajos ponen de manifiesto las diferencias entre las concepciones de los estudiantes para profesor y las directrices actuales (Marks, 1991; Llinares, 1996; Blanco, 1997). El modelo de enseñanza experimentado en la escuela primaria y secundaria ha marcado sus concepciones sobre diversos aspectos de la matemática y de su enseñanza-aprendizaje, como el contenido matemático escolar, los objetivos de la enseñanza de las matemáticas, el currículo matemático, el tipo de tareas a desarrollar, y sobre uno mismo en relación con la educación matemática. 
Además, estas concepciones se van estabilizando y haciéndose resistentes a los cambios conforme avanzan en niveles educativos, y condicionarán el uso que hagan de ellas, bien como ciudadanos o como profesores (Marks, 1991; Gómez-Chacón, 2000).

Para Ponte (1992) y González (1995), las concepciones son una especie de lente o de filtro que los estudiantes utilizan, consciente o inconscientemente, para filtrar, y en ocasiones bloquear (Ponte, 1992), los contenidos de la didáctica de las matemáticas de los cursos de formación y para interpretar su propio proceso formativo. Las concepciones disponen y dirigen también sus experiencias docentes durante las prácticas de enseñanza aunque en algunos aspectos existen contradicciones entre las concepciones y las conductas docentes (Mellado, 1996).

Las referencias que los futuros profesores tienen en cuanto fueron alumnos en la disciplina de matemáticas aparecen casi siempre con influencias fuertes y negativas en el proceso de aprender a enseñar (Borrallo, 1995; Fernandes, 1995; Ernest, 2000).

Dentro de las investigaciones que se vienen desarrollando en España sobre la formación de profesores (Llinares, 1998), existen trabajos centrados en los profesores de primaria en formación (Castro y Castro 1992; Llinares, 1993; Azcárate, 1996; Blanco, 1997; Contreras y Climent, 1999; Hernández, Palarea y Socas, 2000; Contreras y Blanco, 2002).

Y, como consecuencia de ello, los investigadores asumen que, para aprender a enseñar matemáticas, se deben considerar las exigencias que proceden de las propias concepciones y conocimientos sobre la matemática, sobre su enseñanza-aprendizaje y todas las influencias externas implicadas en la educación. Todas ellas forman parte del conocimiento profesional, y deberían ser trabajadas en procesos reflexivos de formación partiendo en todos los casos de las concepciones de los estudiantes, pues las mismas, junto con sus conocimientos, van a caracterizar su futuro como profesores de matemáticas.

\section{RECUERDOS, EXPECTATIVAS Y CONCEP- CIONES SOBRE GEOMETRÍA ESCOLAR Y SU ENSEÑANZA Y APRENDIZAJE}

Asumiendo la importancia que el estudio de las concepciones debe tener en los programas de formación inicial, hemos desarrollado una investigación (Barrantes, 2002) en la que el objetivo principal ha sido describir y analizar las concepciones sobre la geometría escolar y su enseñanzaaprendizaje de los estudiantes para maestro.

En el trabajo hemos partido de la hipótesis (Fig.1) de que los recuerdos y las expectativas de los estudiantes nos dan información para caracterizar sus concepciones en el campo de la geometría y su enseñanza-aprendizaje en primaria.

El término recuerdo aparece en el diccionario de la lengua española (RAE, 1992) como «memoria que se hace o aviso que se da de una cosa pasada o de que ya se habló».
Para la psicología, el recuerdo es «una producción de la memoria que conserva el sabor original de la representación del pasado, así como los detalles, los accidentes y la carga afectiva de acontecimiento.» (Enciclopedia de la Psicología y la Pedagogía, 1978, vol. 7, p. 107).

Los recuerdos que estudiamos se encuentran en la memoria a largo plazo, que es el lugar en el que se almacena la información permanente. Hemos de tener en cuenta que a veces los recuerdos son incorrectos, pues la forma en que aprendemos y procesamos la información y el contexto físico y emocional afectan a la mejor o peor recuperación posterior.

Aunque el recuerdo, en contraposición al saber, es una información mucho más pobre, es también organizada y limitada a lo importante. En nuestro caso, el término se empleará, de acuerdo con las definiciones dadas, como estímulo de la memoria a largo plazo de los estudiantes sobre sus experiencias sobre la geometría y su enseñanza-aprendizaje en su etapa como discentes de primaria.

El término expectativa aparece en el diccionario de la lengua española (RAE, 1992) como «cualquier esperanza de conseguir una cosa, si se depara la oportunidad que se desea».

En nuestro caso, esta definición genérica se traduce en una serie de ideas, actitudes, estrategias y posicionamientos sobre distintos aspectos implicados en la enseñanza-aprendizaje de la geometría, que el estudiante considera que serán idóneos para desarrollar una buena actividad profesional.

Utilizamos el término expectativas y no el de perspectivas, ya que éstas últimas incluyen acciones y no solamente disposiciones al acto; además, las perspectivas son específicas de situaciones y no representan concepciones generalizadas.

El término perspectiva es considerado, en Tabachnick y Zeichner (1984), a partir de la definición de Becker y otros (1961, p. 34), como «un conjunto coordinado de ideas y acciones que una persona utiliza en relación con alguna situación problemática».

En nuestro estudio, por tanto, el término expectativa está más identificado con ideas y actitudes hacia la enseñanza-aprendizaje de la geometría -es decir, lo que podíamos denominar unas perspectivas a priori-, ya que nuestro objetivo no es solamente identificar unas actitudes hacia esta enseñanza sino que los estudiantes expresen sus ideas y reflexionen de una manera genérica sobre aspectos generales como metodología, contenidos, actividades, etc.

El concepto de expectativa se acerca más a lo que Llinares y Sánchez denominan las perspectivas de acción y definen como: «Una serie de expectativas sobre el conocimiento, motivación y conducta del estudiante, así como de posibles estrategias pedagógicas que posiblemente serán efectivas para comunicar el contenido de los alumnos o manejar la clase.» (Llinares y Sánchez, 1990b, p. 168). 
Figura 1

Cuadro general de la hipótesis de investigación.

\begin{tabular}{|l|l|}
\hline $\begin{array}{l}\text { HIPÓTESIS. Los recuerdos y las expectativas de los estudiantes nos dan información con } \\
\text { el fin de caracterizar las concepciones de los estudiantes para maestro en el campo de la } \\
\text { geometría y su enseñanza-aprendizaje en primaria. }\end{array}$ \\
\hline $\begin{array}{l}\text { RECUERDOS } \\
\text { Perbalizables } \\
\text { de aceptación o rechazo }\end{array}$ \\
$\begin{array}{l}\text { EXPECTATIVAS } \\
\text { Verbalizables y explícitas } \\
\text { Plano de los deseos }\end{array}$
\end{tabular}

Finalmente, el significado del término concepción ha sido ya suficientemente tratado en diferentes estudios (Thompson 1992; Pajares, 1992; Mellado, 1996; García, 1997; Flores, 1998; etc.). A partir de ellos, y teniendo en cuenta una amplia variedad de matices, utilizamos el vocablo concepción refiriéndonos, en términos de Thompson, a una estructura mental de carácter general, que incluye «creencias, conceptos, significados, reglas, imágenes mentales y preferencias, conscientes o inconscientes» (Thompson, 1992, p. 132).

El enfoque de nuestro estudio también sigue las directrices más específicas sobre la idea de concepción que Carrillo (1998) y Contreras (1999) utilizan en sus trabajos de tesis: «conjunto de creencias y posicionamientos que el investigador interpreta que posee el individuo, a partir del análisis de sus opiniones y respuestas a preguntas sobre su práctica». (Carrillo, 1998, p. 42). Sin embargo, en nuestro caso las opiniones y respuestas lo serán sobre sus recuerdos y expectativas.

También hemos tenido en cuenta la distinción que hace Thompson (1992) entre concepciones y conocimiento para poder hacer una valoración más rigurosa de las primeras. Así, «una característica de las concepciones es que pueden ser consideradas desde distintos grados de convicción y no son consesuadas» (Thompson, 1992, p. 129), mientras que el conocimiento debe satisfacer condiciones de validez.

Los conocimientos de los estudiantes sobre esta etapa de su vida escolar pueden ser fácilmente explicitados por éstos. No ocurre así con las concepciones que son implícitas y difíciles de mostrar. Por ello, a partir de la información que los estudiantes nos den sobre los recuerdos que producen sentimientos de conformidad o rechazo y sobre las expectativas que son más fácilmente verbalizables y se mueven en el plano de los deseos, queremos obtener información sobre sus concepciones relativas a la geometría y a su enseñanza-aprendizaje.

Nuestra hipótesis considera que, cuando los recuerdos son positivos, el estudiante genera una serie de con- cepciones que redundan en expectativas de enseñanzaaprendizaje similares a las recordadas. También, cuando los recuerdos no son positivos, se produce un sentimiento de rechazo que hace que el alumno conciba una serie de expectativas diferentes a sus recuerdos.

Así, pues, el análisis de esos recuerdos y de esas expectativas que son más fácilmente explicitadas por los estudiantes pueden llegar a darnos información sobre cuáles son las concepciones.

\section{METODOLOGÍA DE LA INVESTIGACIÓN}

De acuerdo con la hipótesis y el objetivo formulado, la investigación se ha llevado a cabo con estudiantes para maestro de la especialidad de primaria que no habían recibido todavía ninguna instrucción sobre la geometría y su enseñanza-aprendizaje en el ámbito de la educación matemática. En estas condiciones, el estudio se realizó con estudiantes para maestro durante los cursos 19961997 y $1999-2000$.

En el trabajo hemos partido de un sistema de categorías y subcategorías elaborado a priori teniendo en cuenta las propuestas curriculares actuales y los trabajos específicos sobre la didáctica de la geometría. Dada la amplitud del tema, decidimos limitarnos a categorías relacionadas con la enseñanza-aprendizaje de la geometría, no considerando otras relacionadas con los conocimientos de la materia, conocimientos pedagógicos o del contexto, que serán objeto de posteriores trabajos.

Las categorías finales fueron:

1) GE - Geometría escolar y su enseñanza

2) $\mathrm{CO}$ - Contenidos escolares de geometría

3) ME - Metodología en la geometría escolar

4) MA - Materiales en la geometría escolar

5) RE - Recursos en la geometría escolar

6) AC - Actividades de geometría escolar 
7) AP - Aprendizaje en la geometría escolar

8) PA - Papel del alumno

9) PM - Papel del maestro

10) EV - Evaluación en la geometría escolar

Dado que nuestro objetivo es fundamentalmente descriptivo-interpretativo, hemos optado por una metodología cualitativa, rica en descripciones y explicaciones de los procesos que ocurren en contextos locales, la cual nos aporta datos de los recuerdos y expectativas de los estudiantes desde el interior del grupo y desde sus propias ideas. De manera complementaria hemos cuantificado algunos resultados para reafirmar o no la significación e influencia de los mismos.

Hemos puesto el énfasis en el lenguaje, en la interpretación de los hechos humanos y en la toma del punto de vista del informante para comprender en, más profundidad, los acontecimientos tal como los viven los estudiantes.

Para ello, hemos utilizado un método no interactivo basado en dos cuestionarios de preguntas abiertas donde plasmar sus recuerdos como discentes y sus expectativas como futuros maestros que impartirán geometría en primaria. Éstos fueron validados por un grupo de investigadores expertos y por una muestra homogénea al grupo a investigar que nos permitió negociar el significado de cada una de las expresiones contenidas para adecuarlas a los objetivos de la investigación. Ambos cuestionarios tenían referencias similares sobre sus experiencias como alumnos y sobre sus expectativas para poder compararlas y explicitar mejor sus concepciones.

Con posterioridad a los cuestionarios, hemos utilizado un método interactivo del tipo grupo de discusión que se trata de una «una técnica no directiva que tiene por finalidad la producción controlada de un discurso por parte de un grupo de sujetos que son reunidos, durante un espacio de tiempo limitado, a fin de debatir sobre determinado tópico propuesto por el investigador.» (Gil, 1992-93, p. 201). La utilización de estos dos métodos nos permite acceder a los procesos internos de los estudiantes ayudándoles a verbalizar sus recuerdos y sus expectativas sobre la geometría escolar mediante sus pensamientos, sus emociones o explicando sus decisiones.

Los grupos de discusión han sido utilizados en otras disciplinas y comienzan a ser empleados en la investigación educativa. Una de las características de estos grupos es establecer y facilitar un debate más que entrevistar al grupo (Watts y Ebbut, 1987), y tiene la ventaja sobre la entrevista individual de minimizar el aspecto intimidador, ya que las personas que comparten un problema estarán más dispuestas a hablar con otras del mismo problema (Lederman, 1990). Los resultados son mejores que los obtenidos a partir de individuos aislados debido a que los productos de las situaciones sociales surgen de la interacción social (Persico y Heawey, 1986). Nuestra experiencia como docentes nos dice que los estudiantes, al comienzo de su formación, no son buenos informantes sobre los temas que vamos a tratar, pues no están acostumbrados a dialogar sobre estos aspectos y tienen bastantes problemas con el léxico específico de educación. En este sentido, Gil afirma: «Los grupos de discusión producen un tipo de datos que difícilmente podría obtenerse por otros medios, ya que configuran situaciones naturales en las que es posible la espontaneidad y en las que, gracias al clima permisivo, salen a la luz opiniones, sentimientos, deseos personales que en situaciones experimentales rígidamente estructuradas no serían manifestados.» (Gil, 1992-93, p. 210).

Para la selección de los individuos de estudio mediante los grupos de discusión, analizamos uno a uno los cuestionarios. Del análisis, observamos que en sus recuerdos no había gran diferenciación; sin embargo, el análisis del segundo cuestionario sobre las expectativas mostraba diferencias significativas que daban lugar a una segmentación de la población en tres grupos de estudiantes diferenciados por una serie de características comunes.

En el primer grupo, la mayoría de sus respuestas mostraban unas ideas y expectativas cercanas a la tendencia tradicional o tecnológica. En el segundo, en cambio, sus ideas eran cercanas a tendencias a priori más innovadoras, es decir, con rasgos espontaneistas o investigativos (Carrillo, 1998; Contreras, 1999; Climent, 2002). Por último, un tercer grupo mostraba en sus declaraciones respuestas a veces contradictorias y que no clasificaban al individuo dentro de los dos grupos formados anteriormente. No había un número significativo de respuestas que les encasillaran en una de las dos tendencias.

Seleccionamos, de cada uno de los tres grupos, cuatro individuos, tras volver a repasar las cuestionarios, para asegurarnos de que los seleccionados eran buenos informantes. El número de personas seleccionadas para cada grupo estaba cerca de los mínimos recomendados para este tipo de estudio; no queríamos correr el riesgo de ver ahogada la interacción y no producir un diálogo lo suficientemente activo (Folch-Lyon y Trost, 1981).

Nuestro papel en los grupos de discusión se limitó a plantear una cuestión, provocar el deseo de discutirla y catalizar la producción del discurso deshaciendo bloqueos y controlando su desarrollo para que se mantuvieran dentro del mismo tema. Procuramos que nuestra intervención fuera mínima para garantizar que la información recibida no había sido filtrada por nuestras reticencias.

Por ello, antes de las intervenciones preparamos una lista de tópicos que deseábamos abordar de acuerdo con las categorías en los cuales nos interesaba corroborar algunas cuestiones y profundizar sobre otras que no habían quedado suficientemente claras en el estudio previo de los cuestionarios.

Para la recogida de los datos utilizamos una grabadora, lo que permitió contar con la sesión completa al llevar a cabo el análisis, ya que «la experiencia ha demostrado que el inicial efecto inhibidor de la grabadora desaparece tras un breve período de tiempo.» (Folch-Lyon y Trost, 1981, p. 448). 
Las sesiones, de una hora aproximadamente de duración, eran suspendidas cuando considerábamos que toda la información sobre los temas a tratar había sido recogida. Llegado un momento, todas las declaraciones de los estudiantes eran reiterativas y no añadían nada nuevo.

Una vez realizada la experiencia, los mismos estudiantes se encargaron de transcribirla. Esto permitía que, al estar implicados en el proceso, anotaran en la transcripción no solamente lo discutido sino todas las observaciones, incluidas las de tipo actitudinal, que ellos descubrieran o recordaran que se habían producido durante la interacción grupal. Registraban las convenciones prosódicas, es decir, las variaciones de tono, intensidad y cantidad de voz, los silencios, etc. La utilización de la grabadora durante las sesiones fue básica para realizar este tipo de anotaciones.

\section{Tratamiento inicial de la información recogida en los cuestionarios}

El elemento básico para el análisis de cuestionarios y grupos de discusión fueron las unidades de análisis que se definen como palabras o conjuntos de ellas procedentes de las respuestas, que tienen significado en relación a los objetivos de la investigación. Las unidades de análisis constituyen un fragmento de texto de unidad variable, dependiendo de la extensión con que se hable del recuerdo o expectativa implicada. Puede ser una oración o un conjunto de oraciones que no tienen por qué coincidir con las respuestas o intervenciones individuales de los estudiantes.

A partir de esas unidades de análisis, en el primer cuestionario se elaboran las ideas núcleo que se definen como: «Una serie de principios, fundamentos o ideas básicas a través de las cuales apoyar y articular los sistemas conceptuales de los estudiantes para maestro.» (Llinares y Sánchez, 1990b, p. 168).

En el segundo cuestionario obtendríamos sus expectativas, que son descripciones de acciones docentes que serían deseables para conseguir una buena enseñanza de la geometría.

En el análisis intervienen también razones como: «Declaraciones verbales, argumentos, que pueden apoyar el establecimiento de las ideas núcleo y que también se utilizan para describir la conexión entre las ideas núcleo y las perspectivas de acción.» (Llinares y Sánchez, 1990b, p. 168).

Éstas, relacionadas con las ideas núcleos o con las expectativas, son afirmaciones que apoyan por qué se mantienen determinados principios o ideas fundamentales.

El estudio conjunto de las ideas núcleo y las expectativas, reforzadas por las razones, realizado en los cuestionarios y en los grupos de discusión, nos daría información sobre las concepciones de los estudiantes (Fig. 2).
Cuestionario sobre recuerdos Grupos de discusión Referencias

Cuestionario de expectativas Grupos de discusión Referencias

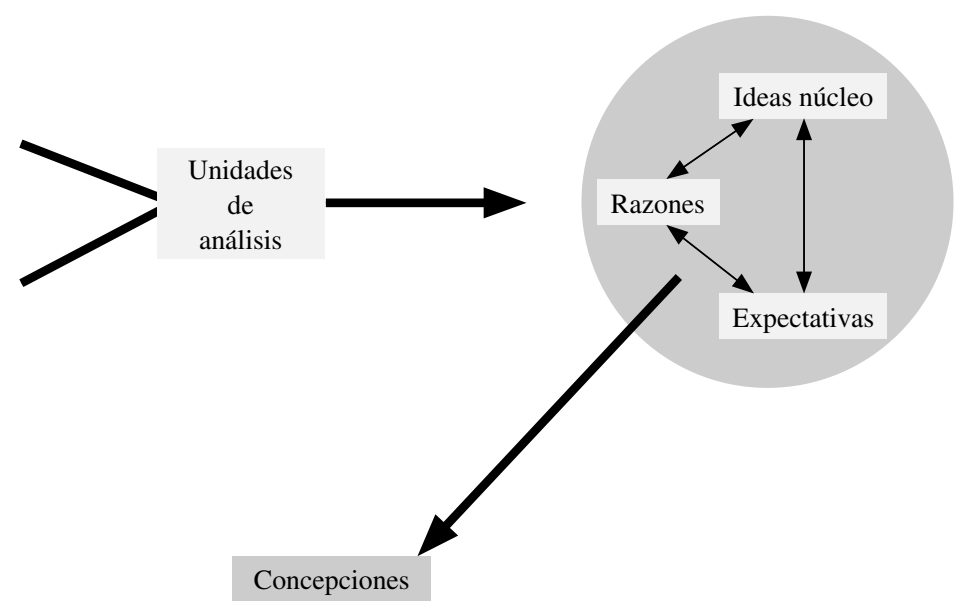


Las fases que hemos considerado para el análisis de los datos en ambos instrumentos son las siguientes:

a) Lectura global de los textos buscando una convergencia de las distintas respuestas de cada ítem o de las transcripciones de los grupos de discusión que nos permitiera iniciar un análisis más sistemático. En el margen de los textos, realizábamos anotaciones buscando su posible relación con alguna categoría o subcategoría, o bien las ideas que más se repiten para su uso posterior.

b) Segmentación en unidades de análisis y su agrupamiento según las categorías y subcategorías establecidas en cada uno de los cuestionarios y grupos de discusión. Esto posibilita la obtención de las ideas núcleo y expectativas que serán apoyadas por diferentes razones.

c) Análisis por separado y luego conjunto de los datos obtenidos en los cuestionarios y en los grupos de discusión, tratando de conocer hasta qué punto los resultados de los grupos de discusión coinciden con los obtenidos en los cuestionarios.

En estos análisis, aunque no es prioritario, está siempre presente un estudio cuantitativo paralelo realizado sobre las frecuencias en las que aparecen las unidades de análisis. Este estudio complementa los resultados obtenidos por la vía cualitativa, pues nos da información sobre qué ideas o expectativas son las que se presentan con mayor frecuencia y qué concepciones y qué tendencia educativa son las más arraigadas.

d) Una fase final de este análisis cualitativo consistirá en verificar los resultados del mismo, es decir, aportar argumentos o realizar comprobaciones que permitan defender que los resultados obtenidos son ciertos. La validez de los resultados de un estudio la entendemos como conectada a la validez de los datos.

En nuestro estudio, para una mayor validación, el análisis conjunto ha sido comparado con documentos e investigaciones del campo de la enseñanza-aprendizaje de las matemáticas o en particular de la geometría (Morales, 1990; NCTM, 1991, 1995; Clements y Battista, 1992; Pérez, 1994; Guillén, 1997; Huerta, 1997; Barrantes, 1998; Contreras y Climents, 1999, Carrillo, 2000; Blanco, 2001; Hernández, Palarea y Socas, 2001, entre otros). De esta manera, se resaltan algunos resultados que son relevantes en la educación geométrica y otros generales de la educación matemática.

\section{La geometría escolar es difícil y difícil de enseñar en la escuela}

Para comprender el trabajo realizado vamos a poner un ejemplo concreto de cómo hemos ido obteniendo las concepciones de los estudiantes para maestro. En este caso, en relación con la primera categoría sobre la geometría escolar y su enseñanza.

En el cuestionario de recuerdos se formulaban diferentes preguntas acerca de los recuerdos de los estudiantes sobre la geometría escolar. Una de ellas, relacionada con la dificultad de la geometría, decía:
«¿Te parecía más difícil la geometría que otras partes que estudiabas de las matemáticas?»

Observamos que una amplia mayoría admitía la mayor dificultad de la geometría. De entre las respuestas dadas, señalamos diferentes unidades de análisis, que asociamos a dicha pregunta:

Todas las partes de la geometría.

La geometría era la parte más difícil para mí.

Otras unidades nos daban información más concreta:

En educación primaria vi muy poco de geometría.

No siempre se da.

Esta idea de poca dedicación a la geometría se llevó a los grupos de discusión y, del debate, entresacamos algunos párrafos:

D.N.: Los temas de geometría eran más cortos y se dan al final o no se daba.

D.C.: La parte de geometría se da con más prisa. Muchas veces no se daban las fórmulas del todo.

F.: En $6^{\circ}$, no llegábamos.

Así pues, una de las razones que justificaban la dificultad de la geometría es la poca dedicación en el periodo escolar. En los textos, buscábamos más razones que nos permitieran comprender sobre la dificultad de la geometría que nos señalaban los estudiantes en las respuestas del cuestionario de recuerdo:

La dificultad está en las fórmulas, había que memorizarlas, y en los problemas.

En los libros de texto sus contenidos están al final.

Y, al mismo tiempo, encontramos algunas ideas núcleo que habíamos inducido a partir de otras unidades de análisis:

Se dedicaba más tiempo a los temas numéricos, que son más fáciles.

El análisis conjunto de todos estos elementos nos permitía elaborar una nueva idea núcleo correspondiente a la primera categoría: «La geometría es más difícil que otras partes que estudiábamos de las matemáticas escolares.»

En la pregunta correspondiente al cuestionario de expectativas de enseñanza-aprendizaje de la geometría escolar encontramos las siguientes razones relacionadas con el mismo tema:

Una materia muy teórica o abstracta.

Complicada de comprender.

Se necesita una mayor capacidad de razonamiento.

Dificultad de memorizar las fórmulas.

Dificultad de los problemas.

En los grupos de discusión se añade que la dificultad de la enseñanza-aprendizaje de la geometría es debido al desconocimiento de los contenidos y la metodología experimentada:

Ra: Cuando tengamos una serie de conocimientos, como a nosotros 
nos lo han enseñado tan mal, pienso que utilizaremos otros métodos o más recursos o, no sé, otra metodología para que los niños lleguen a comprenderla mucho mejor. . . Yo creo que la vemos difícil por como nos la han enseñado, no porque sea difícil. (Grupo 3)

M A: (...) ahora mismo para mí si sería difícil porque yo no me acuerdo de las fórmulas ni nada de eso, pero una vez que ya las sabes y además tienes claro qué tienes que enseñar y cómo enseñarlo, yo pienso que no. (Grupo 2)

De estas razones podemos inducir la expectativa: «La geometría será difícil de enseñar en la escuela.»

El estudio revela que los estudiantes conciben la geometría como una materia difícil influidos por las condiciones desfavorables (poca dedicación, impartida al final del curso...) en las que la aprendieron. Esta idea núcleo junto con el poco dominio que los estudiantes tienen sobre el contenido, metodología y actividades apropiadas hace que, además, en sus expectativas vislumbren dificultades en su actividad como profesores de matemáticas cuando tengan que enseñar geometría.

La idea núcleo y la expectativa considerada nos permite enunciar una concepción de los estudiantes para profesores sobre la enseñanza de la geometría escolar que se correspondería con la primera categoría:

«La geometría escolar es difícil y difícil de enseñar en la escuela.»

\section{RESULTADOS DE LA INVESTIGACIÓN}

Como resultado global podemos afirmar que la tendencia general serían estudiantes en los que sus recuerdos sobre la geometría y su enseñanza-aprendizaje es el factor más importante que influye en sus concepciones, pero que no desean ser imitadores de sus maestros, pues intuyen que hay una cultura de enseñanza-aprendizaje distinta que puede ser aplicada, aunque apenas la conocen ni la han experimentado. Esto hace que sus recuerdos tengan más peso en sus concepciones que sus expectativas.

A modo de resumen presentamos algunos resultados relevantes obtenidos en relación con las categorías establecidas.

\section{Geometría escolar y su enseñanza-aprendizaje}

La finalidad de la geometría es su utilidad en la vida ordinaria. Para algunos estudiantes, también, la finalidad es simplemente adquirir conocimientos, bien como cultura general, porque es una parte de las matemáticas y todas son importantes, o como base para otros conocimientos o de aplicación a la vida real, aunque esto último es más un recurso dialéctico, ya que no saben dar situaciones concretas que ejemplifiquen su idea.

A lo largo de todo el estudio se descubre que hay una disociación entre su cultura matemática y la enculturación matemática de las propuestas curriculares actuales.

\section{Contenidos escolares de geometría}

Los estudiantes tienen lagunas de conceptos de geometría escolar; algunos no conocen ni el contenido básico. Los contenidos que declaran conocer mejor son los relacionados con la geometría plana. Han trabajado menos la geometría espacial y apenas conocen los temas de isometrías. Estos últimos son olvidados en sus propuestas didácticas.

Para ellos, el tema de la medida es el más importante, aunque lo consideran dentro del campo numérico, debido a la enseñanza recibida y por la concepción de aplicabilidad de la geometría en la resolución de problemas y en la vida ordinaria.

Todo esto hace que los temas numéricos, que son a los que más tiempo dedicaba el maestro, sean considerados más asequibles y más importantes en el contexto de la enseñanza-aprendizaje. Así, en sus expectativas, estos temas son prioritarios y serán los temas que enseñen si, en los centros de formación, no hay actuaciones adecuadas que sean capaces de modificar estas concepciones.

También la influencia de sus conocimientos y experiencias les hace concebir que la geometría plana es más fácil que la geometría espacial; por tanto es más importante, y su enseñanza es básica.

\section{Metodología en la geometría escolar}

Los estudiantes conciben que la geometría se debe enseñar de la misma forma que las otras partes de las matemáticas, salvo en el tema de las figuras, pues el alumno las tiene que manipular y por ello es el único que consideran motivante. Los estudiantes muestran que tienen una gran experiencia sobre metodologías de tendencia tradicional y tecnológica y una escasa o nula experiencia en otras metodologías. Cuando quieren mostrar ideas más innovadoras en sus expectativas, se fundamentan en las ideas teóricas adquiridas en las materias de pedagogía o psicología que cursan en la facultad o en su propia creatividad.

La falta de conocimiento de contenidos y de estrategias metodológicas es un gran inconveniente para que los estudiantes den significado al contenido didáctico y hace que lo conciban como algo innecesario y vacío.

\section{Materiales, recursos y actividades}

Recuerdan que en geometría utilizaban materiales (figuras de madera y instrumentos para dibujar), aunque de forma esporádica, por lo que conciben que, en un principio, son motivantes por sí mismos y no por las actividades que se pueden realizar con ellos.

Las actividades consistían en meras construcciones o dibujos sin ningún aprovechamiento didáctico posterior. Además, estas actividades no se planteaban desde la geometría sino desde otras materias como dibujo o plástica. Conciben que no son actividades para aprender geome- 
tría, sino propias de otras materias y, por tanto, la mayoría no las consideran prioritarias en sus expectativas.

Igualmente, aunque la mayoría tiene presente en sus expectativas la relación con la vida cotidiana, ésta se reduce a conocer las formas de algunos objetos y a resolver problemas del libro que hablan de aspectos cotidianos, sobre todo de medidas. Algunos estudiantes conciben esta relación como una actividad final y de refuerzo. En general es una relación artificial que es discordante con la concepción actual de partir de situaciones problema para llegar a la geometría.

La palabra actividad es, para los estudiantes, sinónimo de resolución de ejercicios y problemas tipo, en los que toda la complicación consiste en saber la fórmula que hay que aplicar. No muestran mucho interés por otra clase de actividades como el manejo de figuras o realización de dibujos que son las que conocen.

Las actividades geométricas están directamente extraídas del libro de texto y suelen ser de estudio de elementos de las figuras, clasificación y de medida. Éstas últimas se conciben dentro de las limitaciones algebraicas, simbólicas y formales. Con las mismas concepciones que sus maestros, los estudiantes derivan el estudio de la geometría al mundo de la medida y le dan poca consideración a los análisis y estudios basados en las figuras.

\section{Aprendizaje}

Los estudiantes conciben el aprendizaje basado principalmente en la explicación y la práctica. Para éstos, en general, los alumnos aprenden los conceptos geométricos mediante la explicación del maestro. Posteriormente, en las actividades, se observa que el alumno ha aprendido cuando es capaz de resolver los distintos ejercicios y problemas tipo que incluyen uno o varios conceptos.

La metodología clásica de la que proceden les hace considerar que hay dos aprendizajes distintos: por una parte, los conceptos geométricos, definiciones, propiedades, etc., lo que denominan la teoría y, por otra, la resolución de cada uno de los tipos de problemas, que son necesarios aprender para superar la evaluación. Por supuesto, por las consecuencias que conlleva, este segundo aprendizaje es el más importante.

Entienden que no es recomendable el aprendizaje memorístico y que primero debe ser la comprensión y después la memorización. El concepto de comprensión lo basan en las explicaciones que pretenden desarrollar, aunque algunos consideran que se alcanza cuando se saben aplicar los contenidos en los ejercicios o problemas. Estas concepciones surgen como reacción contraria a sus experiencias, en las que el aprendizaje era básicamente memorístico.

Los estudiantes muestran también grandes carencias con respecto a las relaciones con el maestro o con los propios compañeros. Recuerdan que con el maestro se limitaban a preguntar y resolver las actividades en la pizarra. La resolución de actividades era principalmente individual, salvo algunas actividades de construcción de figuras que se hacían en grupo.
El recuerdo de estas experiencias genera en los estudiantes una dimensión afectiva de acercamiento a los alumnos que aparece en varias categorías. Así, algunos consideran que en el aprendizaje hay que tener en cuenta el interés, la participación o el esfuerzo del alumno. En sus expectativas, la mayoría estima más importante tener en cuenta los intereses de los alumnos que los conocimientos programados, mostrando un deseo de trasladar el centro del aprendizaje al alumno.

La agrupación para el aprendizaje la conciben de una manera mixta, individual y en grupos pequeños, aunque en realidad dan más importancia al trabajo individual que en grupo. Esta importancia esta motivada por la falta de experiencias de actividades en grupos y no porque consideren que el trabajo en grupo no sea adecuado.

\section{Evaluación}

La evaluación es la categoría en la que los estudiantes muestran de una manera más acusada la influencia de sus recuerdos y sobre la que están más desinformados. El examen es el elemento más importante de la evaluación, por encima de las actividades en el aula o los aspectos actitudinales. Sin embargo, que sea considerado el elemento más importante no significa que sea el que estimen más idóneo para realizar las evaluaciones de sus alumnos.

Los estudiantes afirman en sus expectativas que les gustaría que la evaluación se basará más en la observación del proceso de aprendizaje que en resultados de exámenes. La influencia de sus recuerdos les hace optar por una evaluación mixta en la que se tendrá en cuenta ambos aspectos. Al final y al realizar un análisis más completo de las ideas de los estudiantes, prevalece el examen sobre los demás elementos a considerar en la evaluación.

La concepción de cómo debe ser el examen no tiene modificaciones con respecto a sus recuerdos. Los estudiantes están plenamente convencidos de que los exámenes deben ser prácticos y los problemas idénticos a los hechos en clase pero con los datos cambiados. Conciben que de esta manera el alumno debe comprender el problema y así evitarán su aprendizaje de memoria. Sólo algunos estiman que pondrían algunas preguntas de teorías pero en menor proporción que problemas.

\section{CONCLUSIONES FINALES}

En la década de los setenta, el auge que supuso la matemática moderna hizo que la geometría, que hasta esos años había sido una materia importante, pasase a ser una materia escolar de segundo término, ocupando los últimos capítulos de los libros de texto, a los que la mayoría de las veces el maestro no prestaba atención.

Esta circunstancia dio lugar a que los estudiantes para maestros llegaran a los centros de educación con un conocimiento casi nulo de la geometría y sin apenas referentes sobre su enseñanza-aprendizaje. La formación 
posterior que recibieron como estudiantes para maestro estaba más relacionada con otros temas, como el numérico, que con la geometría y su enseñanza-aprendizaje.

Actualmente estas circunstancias deberían haber cambiado como influencia de las propuestas curriculares aprobadas en la década de los noventa (MEC, 1992). Sin embargo, nuestro estudio nos muestra, a pesar de los esfuerzos de los investigadores por presentar nuevos métodos, recursos o materiales sobre enseñanza de la geometría, que muchos estudiantes siguen llegando a las facultades con las mismas experiencias, falta de conocimientos y concepciones sobre esta materia y su enseñanza que hace unos años, lo que indica que se sigue enseñando igual que antes de tales reformas.

Podemos observar que las experiencias clásicas vividas hacen que no tengan vivencias sobre cómo aprender de una forma constructiva mediante la acción, y que no utilicen otros materiales y recursos distintos a los tradicionales. Su falta de estrategias metodológicas y de experiencias les hace concebir que la preparación de actividades de relación de la geometría con las otras matemáticas, con la vida ordinaria o con otras materias, es dificultoso y depende de la imaginación del maestro más que de una buena preparación. Es decir, sus concepciones están lejos de la utilización de diferentes materiales y recursos y de la realización de actividades orientadas a que los alumnos comprendan la geometría, dándole sentido en sí misma, mediante la resolución de problemas en la línea de las orientaciones actuales.

Dada esta situación, consideramos que debemos hacer una reflexión crítica sobre el trabajo en los centros de formación de profesores que posibilite una influencia en la realidad escolar del nuevo enfoque que sobre la geometría escolar se viene proponiendo en las propuestas curriculares desde hace ya algunos años.

\section{REFERENCIAS BIBLIOGRÁFICAS}

AZCÁRATE, P. (1996). Estudio de las concepciones disciplinares de futuros profesores de primaria en torno a las nociones de la aleatoriedad y probabilidad. Granada: Comares.

BARRANTES, M. (ed.) (1998). La geometría y la formación del profesorado en primaria y secundaria. Servicio de $\mathrm{Pu}-$ blicaciones de la UEX.

BARRANTES, M. (2002). «Recuerdos, expectativas y concepciones de los estudiantes para maestro sobre la geometría escolar y su enseñanza-aprendizaje». Tesis doctoral publicada en CD. Cáceres: Servicio de Publicaciones de la Universidad de Extremadura.

BECKER, R., GEER, B., HUGHES, E. y STRAUSS, A. (1961). Boys in white. Chicago: University of Chicago Press.

BLANCO, L. J. (1997). Concepciones y creencias sobre la resolucion de problemas de estudiantes para profesores y nuevas propuestas curriculares. Quadrante. Revista Teorica e de Investigaçao, 6(2), pp. 45-65.

BLANCO, L. J. (2001). Errors in the Teaching/Learning of the Basic Concepts of Geometry. International Journal for Mathematics Teaching and Learning. Revista electrónica editada por Centre for Innovation in Mathematics Teaching at Exerte University, UK and the Mathematics Department at Bessenyei Colleg, Nyiregyháza (Hungría).

BORRALHO, A. (1995). Formaçao de professores de Matemática e resoluçao de problemas, en Mellado, V. y Blanco, L. J. (coords.). La formación del profesorado de ciencias y matemáticas en España y Portugal. Badajoz: Departamento de Didácticas de las Ciencias Experimentales y de las Matemáticas. Universidad de Extremadura.

BROMME, R. (1988). Conocimientos profesionales de los profesores. Enseñanza de las Ciencias, 6(1), pp. 19-29.

CARRILLO, J. (1998). Modos de resolver problemas y concepciones sobre la matemática y su enseñanza de profesores de matemáticas de alumnos de más de 14 años. Algunas aportaciones a la metodología de la investigación y estudio de posibles relaciones. Servicio de Publicaciones. Universidad de Huelva.

CARRILLO, J. (2000). La formación del profesorado para el aprendizaje de las matemáticas. $U N O, 24$, pp. 79-91.

CASTRO, E. y CASTRO, E. (1992). Concepciones sobre área y perímetro; volumen y capacidad detectados en profesores en formación. Revista de Educación, 6, pp. 197-206.

CLEMENTS, D. H. y BATTISTA, M. T. (1992). Geometry and Spatial Reasoning, en Grouws, D.A. (ed.). Handbook of research on Mathematics teaching and learning, pp. 420-464. Nueva York: MacMillan.

CLIMENT, N. (2002). «El desarrollo profesional del maestro de primaria respecto de la enseñanza de la matemática. Un estudio de caso.» Tesis doctoral. Departamento de Didáctica de las Ciencias Sociales. Universidad de Huelva.

CONTRERAS, L.C. (1999). Concepciones de los profesores sobre la resolución de problemas. Servicio de Publicaciones de la Universidad de Huelva.

CONTRERAS, L.C. y CLIMENT, N. (eds.) (1999). La formación de profesores de matemáticas. Servicio de Publicaciones de la Universidad de Huelva.

CONTRERAS, L. C. y BLANCO, L. J. (2002). Aportaciones a la formación inicial de maestros en el área de matemáticas: una mirada a la práctica docente. Cáceres: Servicio de $\mathrm{Pu}$ blicaciones de la Universidad de Extremadura.

ENCICLOPEDIA DE LA PSICOLOGÍA Y LA PEDAGOGÍA (1978). Versión española a cargo de Alonso-Fernández, F. y Cornejo, C.A. Madrid: Sedmay- Lidis.

ERNEST, P. (1989). The knowledge, belief and attitudes of the 
mathematic teacher. A model. Journal of Educational for Teaching, 15(1), pp. 13-33.

ERNEST, P. (2000). Los valores y la imagen de las matemáticas: una perspectiva filosófica. $U N O, 2$, pp. 9-27.

FENNEMA, E. y LOEF, M. (1992). Teacher' Knowledge and its impact, en Grows D.A. (ed.). Handbook of Research on Mathematicis Teaching and Learning, pp. 147-163. Nueva York: MacMillan.

FERNANDES, D. (1995). A perpectiva biográfica e a formação inicial de professores de Matemática: Reflexões a partir de quatro casos, en Mellado, V. y Blanco, L. J. (coords.). La formación del profesorado de ciencias y matemáticas en España y Portugal. Badajoz: Departamento. de Didáctica de las Ciencias Experimentales y de las Matemáticas. Universidad de Extremadura.

FOLCH-LYON, E. y TROST, J. F. (1981). Conducting focus group sessions. Studies in Family Plannig, 12(12), pp. 443-449.

FLORES, P. (1998). Concepciones y creencias de los futuros profesores sobre las matemáticas, su enseñanza y aprendizaje. Granada: Comares.

GARCÍA, M. (1997). Conocimiento profesional del profesor de matemáticas. Conocimiento de función como objeto de enseñanza-aprendizaje. GIEM. Universidad de Sevilla.

GIL, J. (1992-93). La metodología de investigación mediante grupos de discusión. Enseñanza, vol. X-XI, pp. 199-212.

GÓMEZ-CHACÓN, I. M. (2000). Matemática emocional. Los afectos en el aprendizaje matemático. Madrid: Narcea.

GONZÁLEZ, M. (1995). Perspectivas del alumnado de magisterio sobre su formación y su aprendizaje como docente. Revista Española de Pedagogía, 200, pp. 23-43.

GUILLÉN, G. (1997). «El modelo de Van Hiele aplicado a la geometría de los sólidos. Observación de procesos de aprendizaje». Tesis doctoral. Valencia: Universidad de Valencia.

HERNÁNDEZ, J., PALAREA, M.M. y SOCAS, M.M. (2001). Análisis de las concepciones, creencias y actitudes hacia las matemáticas de los alumnos que comienzan la diplomatura de maestro, en Socas, M., Camacho, M. y Morales, A. (eds.). Formación del profesorado e investigación en educación matemática III, pp. 115-125. Universidad de La Laguna.

HUERTA, M. P. (1997). Didáctica de la geometría en la formación de maestros, en Blanco, L. J. y Cruz, M. C. (coords.). Aportaciones al curriculum en la formación inicial de los profesores de primaria en el área de matemáticas. ICE de la Universidad de León.

KOBALLA,T. R. y CRAWLEY, F. E. (1985). The influence of attitude on science teaching and learning. School Science and Mathematics, 85, pp. 222-232.

LEDERMAN, L. C. (1990). Assesing Educational effectiveness: the focus group interview as a technique for data collection. Communication Education, 38, pp. 117-127.

LLINARES, S. (1993). Aprender a enseñar: reflexiones sobre la formación inicial de profesores de matemáticas. Revista de Enseñanza Universitaria, 5, pp. 111-126.
LLINARES, S. (1996). Contextos y aprender a enseñar matemáticas: el caso de los estudiantes para profesores de primaria, en Gimenez, J., Llinares, S. y Sánchez, V. (eds.). El proceso de llegar a ser un profesor de primaria. Cuestiones desde la educación matemática, pp. 13-36. Granada: Comares.

LLINARES, S. (1998). La investigación «sobre» el profesor de matemáticas: aprendizaje del profesor y práctica profesional. Aula, 10, pp. 153-179.

LLINARES, S. y SÁNCHEZ, M. V. (1990a). Teoría y práctica en educación matemática. Sevilla: Alfar.

LLINARES, S. y SÁNCHEZ, M. V. (1990b): Las creencias epistemólogicas sobre la naturaleza de las matemáticas y su enseñanza y el proceso de llegar a ser un profesor. Enseñan$z a, 8, \mathrm{pp} .165-180$.

MARKS, R. (1991): When should teachers learn pedagogical content knowledge? Chicago: AERA.

MEC (1992). Primaria. Área de matemáticas. Madrid: MEC.

MELLADO, V. (1996). Concepciones y prácticas de aula de profesores de ciencias, en formación inicial de primaria y secundaria. Enseñanza de las Ciencias, 14 (3), pp. 289-302.

MORALES, A. (1990). Algunas consideraciones sobre la enseñanza de la geometría elemental. El Guiniguada, 1, pp. 57-66.

NCTM (1991). Estandares curriculares y de evaluación para la educación matemática. Sevilla: Sociedad Andaluza de Educación Matemática «Thales».

NCTM (1995). Assessment standards for school mathematics. Virginia: NCTM. Reston.

PAJARES, F. (1992). Teachers' Beliefs and Educational Research: Cleaning up a Messy Construct. Review of Educational Research, 62(39), pp. 307-332.

PÉREZ, R. (1994). Construir la geometría. UNO, 2, pp. 65-80.

PERSICO, C. y HEAWEY, T.W. (1986). Group interviews: a social methodology for social inquiry. ERIC Document Reproduction Service, núm. ED 275915.

PONTE, J.P. (1992). Concepções dos Profesores de Matemática e Processos de Formação, en Brow, M., Fernandes, D., Matos J. F. y Ponte, J. P. Educação Matemática, pp. 185239. Lisboa: Instituto de Inovação Educacional.

RAE. (1992). Diccionario de la lengua española. 20a. edición.

TABACHNICK, B. R. y ZEICHNER, K. M. (1984). The Impact of the Student Teaching Experience on the Development of Teacher Perspectives. Journal of Teacher Education, nov.- dic., pp. 28-35.

THOMPSON, A. G. (1992). Teachers beliefs and conceptions: A synthesis of the research, en Grouws, D. A. (ed.). Handbook of research on Mathematics teaching and learning, pp. 127-146. Nueva York: MacMillan.

WATTS, M. y EBBUTT, D. (1987). More than de sum of the parts: research methods in group interviewing. British Educational Research Journal, 13(1), pp. 25-34. 